\title{
LA PANDEMIA SILENZIOSA: I BAMBINI RIMASTI ORFANI
}

Una ricaduta pediatrica drammatica del Covid-19, fino a ora poco valutata, è quella dei bambini rimasti orfani a causa dell'epidemia. Si tratta di una vera e propria catastrofe, anche perché lo stato di orfano comporta, oltre che una devastazione emotiva, un aumentato rischio di povertà, istituzionalizzazione e abuso.

È la cosiddetta "pandemia nascosta del 202I", quella a cui si fa riferimento nel rapporto della CDC (U.S. Centers for Disease Control and Prevention) sugli orfani da Covid- 19 e le possibili strategie di intervento'.

La mortalità da infezione da Covid-19 è più elevata nella fascia di età adulta-anziana, e risparmia bambini e adolescenti. Gli interventi e le risorse a livello mondiale si sono focalizzate in primis sulla prevenzione dell'infezione, la ricerca di cure efficaci, la riduzione della mortalità, lo sviluppo e la distribuzione del vaccino. Questo ha distolto l'attenzione dai bisogni dei bambini, e all'improvviso ci siamo resi conto che la pandemia ha avuto tragiche conseguenze anche su coloro che hanno perso un genitore o anche un nonno. In età infantile una perdita del genere comporta bruschi e importanti cambiamenti e ha effetti devastanti a lungo termine sulla salute fisica, mentale, oltre che sul benessere economico.

Se consideriamo la non equa accessibilità al vaccino che esiste nei diversi Paesi del mondo, l'alto tasso di no-vax, lo sviluppo di varianti più contagiose e resistenti e la potenziale riduzione dell'immunità acquisita è verosimile che nei Paesi con più morti da Covid l'immunità "di gregge" non potrà essere raggiunta prima dei prossimi 4 anni. Se queste stime sono veritiere dovremo aspettarci più di 4 milioni di bambini che diventeranno orfani, con effetti a lungo termine che si protrarranno per almeno una decade'.

\section{Gli studi e i numeri}

Lo studio di Hillis e coll. ${ }^{2}$ pubblicato su Lancet lo scorso luglio, basato sui dati di natalità e mortalità in 21 Paesi (che hanno contribuito al $77 \%$ delle morti mondiali per Covid), ha potuto stimare che, a causa della pandemia, dal $\mathrm{I}^{\circ}$ marzo 2020 al 30 aprile 202I I.134.000 bambini e adolescenti < I8 anni sono rimasti orfani di almeno un genitore o hanno perso un nonno tutore; circa I.562.000 hanno subito la perdita di almeno un caregiver primario o secondario (figura parentale) (Box I e Figura I).

II rimanere orfani ha avuto una massima incidenza in Perù ( 10 per mille bambini), Messico, Brasile, Colombia, Sud Africa e Iran (circa 3 per mille), ma è un problema drammaticamente misurabile anche in Paesi come gli USA (I,5 per mille), Argentina e Russia (I per mille). Gli orfani di padre sono stati tre volte di più che quelli di madre.

In questo studio sono state considerate come morti da Covid non solo quelle determinate direttamente dalla malattia nei soggetti infetti, ma anche le morti indirette dovute alle conseguenze della pandemia sulla popolazione generale (ad esempio per la ridotta accessibilità ai Servizi di salute pubblica, in particolare per i malati cronici) ${ }^{2}$.

\section{Box 1 - DEFINIZIONI}

\section{Chi è un orfano?}

Secondo la definizione UNICEF, ogni minore di 18 anni di cui uno o entrambi i genitori siano morti.

Chi è un nonno "tutore"?

Un nonno/a (età dai 60 agli 84 anni) che vive nella stessa casa del minore e si prende cura di lui al posto dei genitori, che sono assenti in casa, ovvero un nonno che funge da genitore.

Chi è un nonno convivente e caregiver?

Un nonno/a (età dai 60 agli 84 anni) o anche un'altra figura parentale che vive nella stessa casa del minore ma insieme ai suoi genitori, e insieme a loro se ne prende cura.

Cosa vuol dire "perdere un caregiver primario"?

Perdere uno o entrambi i genitori, oppure uno o entrambi i nonni tutori.

Cosa vuol dire "perdere un caregiver secondario"?

Perdere un nonno convivente e caregiver.

II più recente studio pubblicato su Pediatrics il 7 ottobre 202 I si è focalizzato sui dati di bambini orfani da Covid negli USA, utilizzando lo stesso metodo di rilevazione e le stesse definizioni di orfani, caregiver primari e secondari ${ }^{3}$.

Dal I ${ }^{\circ}$ aprile 2020 al 30 giugno 202I, più di 140.000 bambini negli USA hanno perso un caregiver primario o secondario. Circa 90.000 di quelli che hanno perso un caregiver primario, e quindi più della metà del totale, fanno parte di minoranze etniche e razziali. Tra i bianchi I/753, tra gli ispanici I/4I2, tra i neri 1/310, tra i nativi americani I//68 ha perso un genitore o un caregiver. Sebbene la maggior parte dei morti per CovidI9 sia stata nella popolazione bianca (5 su 10), la maggior parte degli orfani invece riguarda le minoranze etniche (circa 7 su 10), e si calcola che i bambini nativi americani, neri, ispanici e asiatici abbiano un rischio maggiore da 2 a 4 volte di perdere un genitore o un caregiver, rispetto ai bambini bian$\mathrm{chi}^{3}$.

Le stime sono consultabili liberamente sul sito del London Imperial College (https://imperialcollegelondon.github.iol orphanhood_calculator/\#/country) dove un calcolatore in tempo reale permette di visualizzare e confrontare la situazione di ogni Paese del mondo (Figura 2). Per elaborarle un team di ricercatori internazionali - II Gruppo di Riferimento Globale sui bambini colpiti da Covid-19 - ha utilizzato un complesso sistema di calcolo basato sui dati della mortalità e natalità nazionali, processati con un modello statistico.

Lascia senza parole il dato dell'India che conta a ottobre 2021 (quindi con una finestra temporale che va oltre quella dello studio pubblicato su Lancet) oltre I.800.000 orfani.

Se guardiamo a casa nostra i dati al 12 ottobre 202 I sono di 3500 orfani, 3900 che hanno perso un caregiver primario, 6300 che hanno perso un caregiver primario o secondario. 


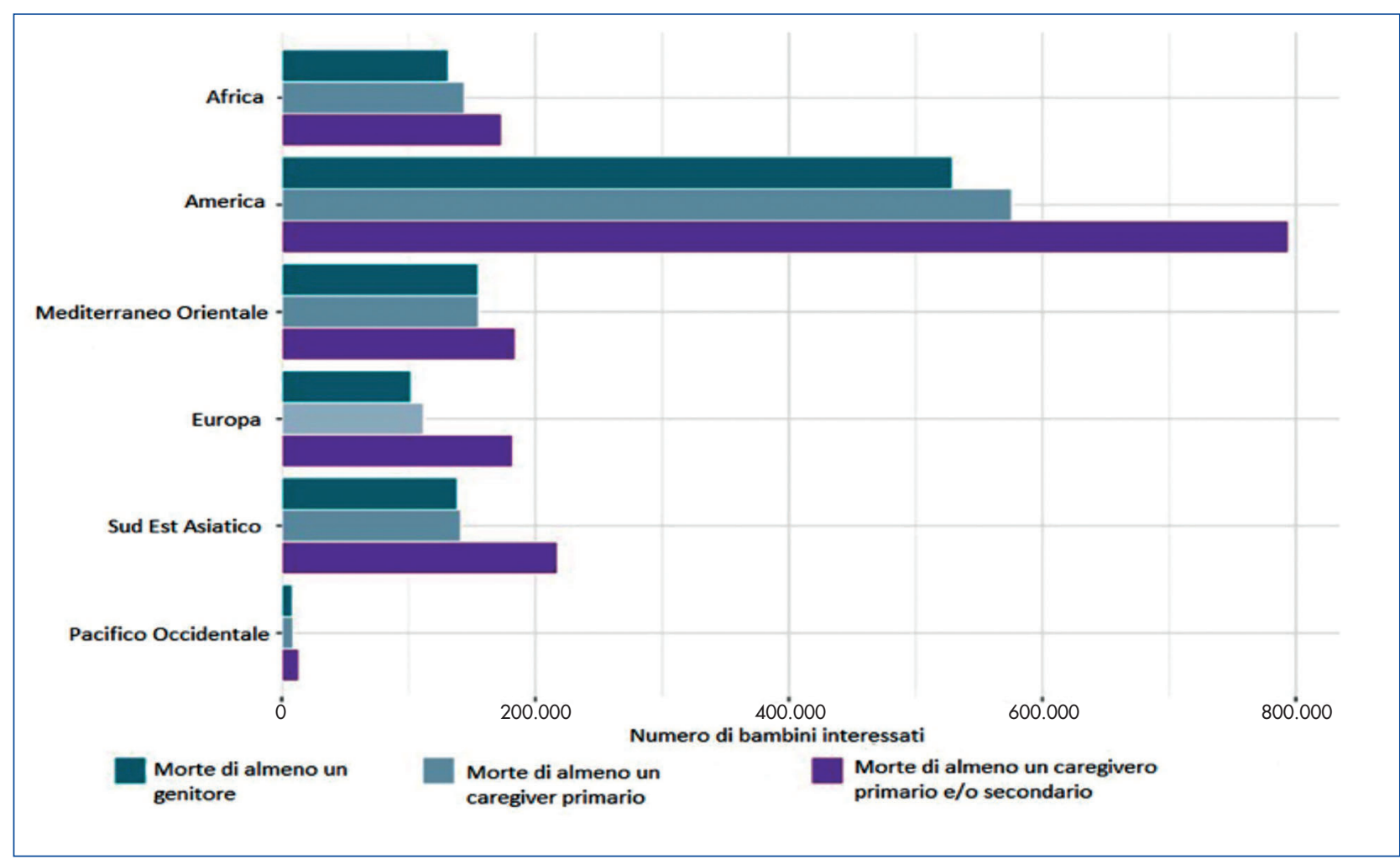

Figura 1. Numero stimato di bambini rimasti orfani da Covid-19 nei diversi continenti (da voce bibliografica 1, modificata).

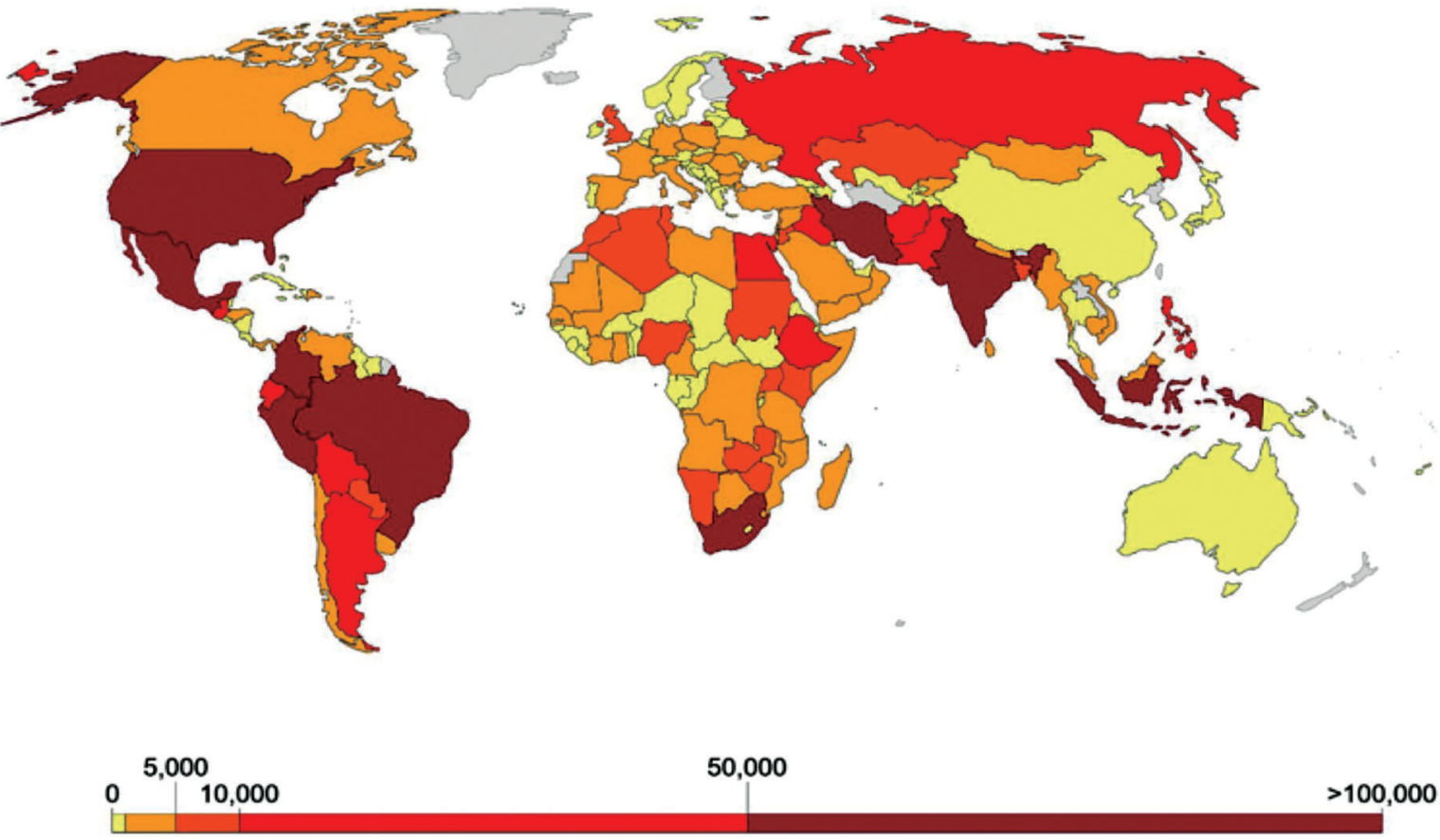

Figura 2. Stima nel mondo dei bambini "orfani" da Covid-19 aggiornata al 4 ottobre 2021. Da: https://imperialcollegelondon. github.io/orphanhood_trends/. 


\section{II commento}

II Covid- I 9 ha messo in evidenza le disparità sociali e di salute nell'incidenza di malattia, nella sua severità e nei suoi esiti, tra i vari gruppi razziali, etnici e geografici. Tali disparità derivano dalla disuguaglianza nei diritti di accesso ai Servizi di salute, dai maggiori problemi di disoccupazione, dalle discriminazioni, dai gap educazionali e dalla maggiore instabilità economica e probabilità di vivere in condizioni precarie.

Circa il $23 \%$ dei bambini americani vive con un solo genitore e perdere questa figura di riferimento significa non avere più nessuno che sia responsabile delle sue cure ${ }^{3}$.

Sempre più frequentemente $i$ nonni vivono all'interno di nuclei familiari multigenerazionali dove si trovano ad avere un ruolo fondamentale nella cura dei nipoti. Si calcola che nel mondo il 38\% dei bambini vive in famiglie allargate insieme ai loro nonni, e questa percentuale sale al $50 \%$ nelle regioni asiatiche. I nonni spesso provvedono in maniera significativa nel supportare finanziariamente i nipoti (in Brasile ad esempio fino al $70 \%$ dei casi) ${ }^{2}$.

Negli USA circa il 10\% dei minori vive in casa con un nonno, e questa percentuale si raddoppia tra i neri, ispanici e asiatici rispetto ai bianchi. Inoltre la maggior parte dei bambini che convivono con i nonni hanno un solo genitore vivo o sono già orfani ${ }^{3}$.

La morte di un familiare stretto per un bambino è uno degli eventi più traumatici che la vita possa riservare. La rapidità con cui il Covid-1 9 causa malattia e morte lascia a disposizione poco tempo per preparare i bambini a un tale trauma. La perdita di un genitore o di un caregiver può causare problemi di salute mentale come ansia, depressione, stress post-traumatico, bassa autostima, con associato un maggior rischio di subire violenza fisica, sessuale e psicologica.

Perdere un genitore $o$ un caregiver si associa inoltre a un aumentato rischio di abbandono scolastico, di suicidio, di patologie a trasmissione sessuale, di gravidanza precoce, di malattie croniche e morte prematura.

Non meno importanti sono i disagi di natura economica, in quanto la pandemia ha aggravato situazioni di povertà preesistente, con ulteriore riduzione della disponibilità dei mezzi di sussistenza, di scolarizzazione, fornitura di Servizi generali e di salute.

Per un bambino che ha perso una figura di riferimento è determinante il nucleo familiare. Ogni bambino ha bisogno di una famiglia: sembra scontato, ma non è così. Per raggiungere a pieno lo sviluppo delle sue capacità fisiche, intellettuali e sociali, ha bisogno di un genitore elo di un caregiver che si prenda cura di lui in maniera stabile. Orfanotrofi, collegi, case famiglia non sono un'alternativa adeguata a lungo termine.

Paesi con più alto tasso di orfani causati dal Covid necessitano di supporto immediato, a livello locale, nazionale e internazionale. Nonostante ci sia una vasta letteratura che dimostri i danni associati all'istituzionalizzazione dei minori (influenzano negativamente lo sviluppo cognitivo, emozionale e sociale, ed espongono i bambini a un maggior rischio di abuso), tali strutture sono purtroppo aumentate anziché diminuire. Questo perché è sicuramente più facile chiudere un bambino in un istituto anziché reintegrarlo in un nucleo familiare disponibile a prendersi cura di lui, ma che va necessariamente sostenuto. I bambini hanno bisogno di una famiglia, e le famiglie hanno bisogno di essere supportate. Se non ci sono familiari che possano prendersi cura del bambino le alternative sono l'affido e l'adozione, ma in queste scelte i minori devono essere coinvolti, e i fratelli e le sorelle non vanno separati. Le risorse dovrebbero essere indirizzate ad assicurare che ogni bambino rimasto orfano abbia una famiglia sicura che deve essere supportata.

Le strategie di intervento sono dunque su più fronti, così riassumibili:

- prevenire la morte dei caregiver accelerando l'accesso ai vaccini, garantendoli equamente anche nei Paesi più poveri;

- programmi e servizi di supporto evidence-based mirati a ridurre il rischio delle conseguenze negative che le perdite di genitori elo nonni possano avere sulla salute mentale e fisica di bambini e adolescenti, rafforzando la loro capacità di ripresa e la loro resilienza;

- far sì che i bambini vittime di questi lutti non siano chiusi in orfanotrofi, ma che siano mantenuti in una famiglia (la propria o adottivalaffidataria se necessario) che deve essere aiutata economicamente, socialmente e psicologicamente;

- garantire l'istruzione scolastica.

È necessario l'intervento dei Governi locali, nazionali, delle Organizzazioni internazionali, con azioni mirate in quelle aree del mondo dove il tasso di orfani causati dalla pandemia è più elevato ${ }^{1-3}$. Si tratta di una prospettiva che ogni pediatra e ogni Istituzione dedicata all'infanzia deve conoscere per favorire interventi comunitari consapevolmente concreti.

Angela Troisi, Federico Marchetti
UOC di Pediatria e Neonatologia
Ospedale di Ravenna, AUSL della Romagna

\section{Bibliografia}

I. U.S. Centers for Disease Control and Prevention (CDC), U.S. Agency for International Devel-opment (USAID), The World Bank, World Health Organization, University of Oxford, University College London, Imperial College London, University of Cape Town. Children: the hidden pan-demic 202I. A joint report of Covid-19 associated orphanhood and a strategy for action. 202I.

2. Hillis SD, Unwin HJT, Chen Y, et al. Global minimum estimates of children affected by Covid-19-associated orphanhood and deaths of caregivers: a modelling study. Lancet 202 I;398(I0298):39|-402. doi: 10.1016/S0140-6736(2I)01253-8.

3. Hillis SD, Blenkinsop A, Villaveces A, et al. Covid-19-associated orphanhood and caregiver death in the United States. Pediatrics 2021 Oct 7;e2021053760. doi: 10.1542/peds.2021-053760 [online ahead of print]. 